\title{
On interpreting the photoelectron spectra of $\mathrm{MgO}^{-}$
}

\author{
Charles W. Bauschlicher, Jr.* and Harry Partridge \\ Mail Stop-230-3 \\ NASA Ames Research Center \\ Moffett Field, CA 94035
}

\begin{abstract}
The ${ }^{2} \Sigma^{+}$and ${ }^{2} \Pi$ states of $\mathrm{MgO}^{-}$and the ${ }^{1} \Sigma^{+},{ }^{1} \Pi$, and ${ }^{3} \Pi$ states of $\mathrm{MgO}$ are studied using the ACPF approach. The computed spectroscopic constants are in good agreement with the available experimental data. The computed Franck-Condon factors and photodetachment overlaps are compared with experiment.
\end{abstract}

\section{INTRODUCTION}

Photoelectron spectroscopy (PES) of anions can reveal a wealth of data about both the neutral and anion. Since the PES selection rules are different from those for electronic transitions between states of neutrals, it is possible to observe neutral states that have forbidden transitions with the ground state. In addition to transition energies, PES can be used to determine reasonably accurate vibrational frequencies. From hot bands, it is often possible to measure anion vibrational frequencies. From a Franck-Condon simulation of the observed spectra, differences in bond lengths between the anion and neutral state can be determined. In principle low-lying anion

\footnotetext{
"bauschli@pegasus.arc.nasa.gov, (650)604-6231 and fax $=(650) 604-0350$
} 
states can contribute to the spectra, and if both anion states form a common neutral state, PES can yield anion excitation energies.

If the neutral states are close in energy, the spectra can become very complicated due to overlapping bands. Low-lying excited anion states can make the interpretation even more complicated. Recently the Johns Hopkins University (JHU) group measured ${ }^{1}$ the photoelectron spectra of $\mathrm{MgO}^{-}$. Their spectra is shown in Fig 1 . The peaks labeled $C, D$, and $E$ are consistent with a progression in the vibrational levels of the ${ }^{1} \Sigma^{+}$state of $\mathrm{MgO}$, while peak $\mathrm{B}$ appears to be a hot band associated with vibrational excitation in the anion. The peaks at higher electron binding energy are consistent with the known ${ }^{2-4}$ low-lying excited states of $\mathrm{MgO}$. The origin of the peaks labeled $A$ is not obvious. The peak separations are inconsistent with vibrational levels in the ${ }^{1} \Sigma^{+}$state and it is not possible to attribute both peaks $A$ and $B$ with vibrational excitation of the anion. If one assumes that $A$ is due to an excited state of the anion forming an excited state of the neutral, one would expect to see a peak associated with the formation of the neutral ground state at lower electron binding energy, which is not observed in experiment. In order to aid in the interpretation of this spectra, ab initio calculations were carried out on $\mathrm{MgO}$ and $\mathrm{MgO}^{-}$. We should note that additional experiments ${ }^{1}$ were carried out at Pacific Northwest National Laboratory and Washington State University (PNNL/WSU). The conditions in the PNNL/WSU experiment were different from those in JHU experiments, and their conclusions $^{1}$ based on an analysis of the experimental work is in agreement with our analysis based on the calculations reported in this manuscript.

\section{QUALITATIVE CONSIDERATIONS}

The ground state of $\mathrm{MgO}$ is known to be ${ }^{1} \Sigma^{+}\left(6 \sigma^{2} 2 \pi^{4}\right)$. The $a^{3} \Pi$ and $A^{1} \Pi$ states are 2620 and $3563 \mathrm{~cm}^{-1}$, respectively, above the $X^{1} \Sigma^{+}$state $\mathrm{s}^{2-4}$. These two $\Pi$ states are derived from the $6 \sigma^{2} 7 \sigma^{1} 2 \pi^{3}$ occupation. These three states are perhaps best 
viewed as arising from $\mathrm{Mg}^{+}\left({ }^{2} S\right)$ and $\mathrm{O}^{-}\left({ }^{2} P\right)$. In the ${ }^{1} \Sigma^{+}$state, the singly occupied $\mathrm{O}^{-} \sigma$ orbital forms a bond with the open-shell $\mathrm{Mg} 3 s$ orbital. In the II states, the $\mathrm{O}^{-}$has a $2 \mathrm{p} \sigma^{2} 2 \mathrm{p} \pi^{3}$ occupation; the $6 \sigma$ bonding orbital is doubly occupied while the $7 \sigma$ antibonding orbital is singly occupied. Donation from the $O 2 \mathrm{p} \pi$ orbital into the empty $\mathrm{Mg} 3 \mathrm{p} \pi$ orbital contributes to the bonding in these states as well as in the ${ }^{1} \Sigma^{+}$ state.

Given the bonding in $\mathrm{MgO}$, it is clear that the best view of $\mathrm{MgO}^{-}$is obtained by considering the $\mathrm{Mg}^{1} S\left(3 s^{2}\right)+\mathrm{O}^{-2} P$ limit. $\mathrm{A}^{2} \Sigma^{+}\left(6 \sigma^{2} 7 \sigma^{1} 2 \pi^{4}\right)$ state arises from singly occupying the $O 2 \mathrm{p} \sigma$ orbital; as in the neutral $\Pi$ states, the bonding $6 \sigma$ is doubly occupied and the antibonding $7 \sigma$ is singly occupied. While this state only has a bond order of $1 / 2$ in the sigma space, the oxygen $\pi$ donation to the empty $\mathrm{Mg} 3 \mathrm{p} \pi$ orbital enhances the bonding, as found for the neutral states. A ${ }^{2} \Pi\left(6 \sigma^{2} 7 \sigma^{2} 2 \pi^{3}\right)$ state of $\mathrm{MgO}^{-}$arises if the $\mathrm{O}^{-} 2 \mathrm{p} \pi$ orbital is singly occupied. This state has no formal $\sigma$ bond and only three $\pi$ electrons for donation to the $\mathrm{Mg}$, and therefore is clearly not the ground state of $\mathrm{MgO}^{-}$.

\section{METHODS}

The $\mathrm{MgO}$ and $\mathrm{MgO}^{-}$potentials are computed using the averaged coupled pair functional ${ }^{5}$ (ACPF) approach correlating the valence electrons, namely the $\mathrm{Mg} 3 \mathrm{~s}$ electrons and the oxygen $2 s$ and $2 p$ electrons. Internal contraction ${ }^{6}$ (IC) is used to reduce the computational effort in the ACPF calculations. The $\mathrm{Mg}$ basis set is the correlation consistent polarized valence quadruple zeta (cc-pVQZ) set of Woon and Dunning, while for oxygen the augmented-(aug) cc-pVQZ set is used ${ }^{7-9}$. A completeactive-space self-consistent-field (CASSCF) approach is used to optimize orbitals. Separate CASSCF calculations are performed for each state; for the $\Pi$ states, the two components are averaged so the $\pi_{x}$ and $\pi_{y}$ orbitals are equivalent. The active space consists of the $\mathrm{Mg}$ 3s and 3p orbitals and the oxygen 2p orbital. All configurations 
in the CASSCF are used as references in the ACPF calculations. The CASSCF/ICACPF calculations are performed using MOLPRO ${ }^{10}$.

The overlap of the $\mathrm{MgO}^{-}$and $\mathrm{MgO}$ wave functions is computed using the sudden approximation. These calculations are performed using the triple zeta (TZ) cc-pV and aug-cc-pV basis sets for $\mathrm{Mg}$ and $\mathrm{O}$, respectively. The same active space is used in the CASSCF calculation, but the orbitals are optimized for the average of the ${ }^{2} \Sigma^{+}$and ${ }^{2} \Pi$ states of $\mathrm{MgO}^{-}$. The overlaps are computed at the configuration interaction level using a first-order wave functions, where one hole is allowed in the inactive (O 2s-like orbital) plus active space, and one particle is allowed in the virtual space. That is, the bra is a first-order wave function for the anion with one electron deleted, while the ket is a first-order wave function for the neutral. This wave function should account for orbital relaxation and therefore obtain a reasonable description of all five states. These calculations are performed using the Molecule-Sweden ${ }^{11}$ program system.

\section{RESULTS AND DISCUSSION}

The computed spectroscopic constants are reported in Table I, along with the experimental results ${ }^{1,2,4}$. Our computed $r_{e}$ values are in good agreement with experiment, being slightly too long, which probably arises mostly from the neglect of core-valence correlation. The difference between theory and experiment for the $\mathrm{MgO}^{-}$ ${ }^{2} \Pi$ state is larger than found for the other states. Since the potential for this state is shallower than the others, we suspect the larger differences arises from a larger uncertainty in both the experimental and theoretical values. Our $\omega_{e}$ values for the ${ }^{1} \Pi$ and ${ }^{3} \Pi$ states agree very well with experiment. The error is slightly larger for the ${ }^{1} \Sigma^{+}$state, which is harder to describe since it is has more multireference character. Our computed $\omega_{e}$ values for $\mathrm{MgO}$ and $\mathrm{MgO}^{-}$essentially agree with the photoelectron results to within the experimental error bars.

Comparing our $\mathrm{MgO}$ and $\mathrm{MgO}^{-}$results with recent calculations, we find that our 
$\mathrm{MgO}$ results are similar to those reported by Thümmel el al. ${ }^{12}$. Our $\mathrm{MgO}$ electron affinity (EA) and $\mathrm{MgO}^{-2} \Sigma^{+} r_{e}$ values are very similar to those reported by Gutsev et al. ${ }^{13}$, and therefore we are very surprised that their $\mathrm{MgO}^{-} \omega_{e}$ value is so large $\left(897 \mathrm{~cm}^{-1}\right)$.

Our $T_{e}$ value for the ${ }^{2} \Sigma^{+}$and ${ }^{2} \Pi$ states of $\mathrm{MgO}^{-}$is in excellent agreement with experiment. This is not unexpected for two states that are well described by a single reference configuration. Our computed electron affinity (EA) is too small, as expected. Since the anion has one more electron than the neutral, the EA tends to increase as the level of theory is improved and more of the electron correlation is recovered. Our ${ }^{3} \Pi{ }^{1} \Pi$ separation $\left(855 \mathrm{~cm}^{-1}\right)$ is in reasonable agreement with experiment $\left(940 \mathrm{~cm}^{-1}\right)$, having an error only slightly larger than found for the separation between the ${ }^{2} \Sigma^{+}$ and ${ }^{2} \Pi$ states of $\mathrm{MgO}^{-}$. Unlike the other states, the ${ }^{1} \Sigma^{+}$state is clearly too high in energy with respect to the $\Pi$ states by almost $1000 \mathrm{~cm}^{-1}$. That is, the error is an order of magnitude larger than found for other states. This is probably due greater complexity in describing this state, and the use of a larger active space would improve the position of the ${ }^{1} \Sigma^{+}$state with respect to the others. However, it is clear that these potential are sufficiently accurate to address the interpretation of the photoelectron spectra.

While it is difficult to compute intensities of the different detachment processes, qualitative insight can be obtained by using the sudden approximation to compute the overlap of the $\mathrm{MgO}^{-}{ }^{2} \Sigma^{+}$and ${ }^{2} \Pi$ state wave functions with those for the neutral states, and these values are reported in Table II. First considering detachment from the ${ }^{2} \Sigma^{+}$state, we note that all three overlaps are smaller than the maximum values (i.e. the value obtained if all of the states were described by a single configuration using the same orbitals) of 1,1 , and 3 , for the formation of the ${ }^{1} \Sigma^{+},{ }^{1} \Pi$, and ${ }^{3} \Pi$ states, respectively. The value for the ${ }^{3} \Pi$ state is larger than the others since there are three ways of removing one of the four $\pi$ electrons to form the ${ }^{3} \Pi$ state, while 
only one way to form the ${ }^{1} \Pi$ state. Clearly removing the electron from the $7 \sigma$ orbital is the only way to form the ${ }^{1} \Sigma^{+}$state. The computed values are smaller than the maximum allowed because the states do not have the same orbitals and all states have some multireference character. However, the computed values are all sizable. For detachment from the ${ }^{2} \Pi$ state, it is clear that the ${ }^{1} \Sigma^{+}$state will not be observed since the overlap is essentially zero. That is, the different orbitals for different states and multireference character that reduces the other values from their maximum, does not yield any significant overlap of the ${ }^{2} \Pi$ and ${ }^{1} \Sigma^{+}$states. The overlaps for the formation of the ${ }^{1} \Pi$ and ${ }^{3} \Pi$ states are large and therefore if the ${ }^{2} \Pi$ state is formed, detachment to form the ${ }^{1} \Pi$ and ${ }^{3} \Pi$ states should be observed.

On the basis of these overlaps, it is clear that only five of the six possible detachment processes in the energy range of the JHU experiment will be detectable. This implies that the peaks labeled "A" in Fig. 1 can be attributed to detachment from the ${ }^{2} \Pi$ sate of $\mathrm{MgO}^{-}$forming the ${ }^{3} \Pi$ state of $\mathrm{MgO}$ even though the formation of the ${ }^{1} \Sigma^{+}$state at lower electron binding energy is not observed.

The Franck-Condon factors and the Franck-Condon factors times the sudden overlap terms are reported in Table III. To account for the known errors in our computed results, we shift the ${ }^{1} \Sigma^{+}$up to agree with the experimental EA, and shift the ${ }^{1} \Pi$ and ${ }^{3} \Pi$ states to agree with the known $\mathrm{n}^{2,3}$ separations. Using these Franck-Condon factors times the overlaps, we generate a synthetic photoelectron spectra assuming an equal population of $v=0,1$, and 2 , levels of the the anions, and a relative population of the ${ }^{2} \Sigma^{+}$and ${ }^{2} \Pi$ states of 10 to 1 . This spectra is shown in Fig. 2. It is in reasonable agreement with experimental spectra in Fig. 1. The individual components are shown below the total spectra. The ${ }^{2} \Sigma^{+}-{ }^{1} \Sigma^{+}$contribution is not spread out enough, for example, peak $\mathrm{D}$ is too small and peak $\mathrm{E}$ is missing in our simulated spectra. The ${ }^{2} \Sigma^{+}{ }_{-}^{3} \Pi$ simulation suggests that we have too much vibrational excitation for the anion, for example, the $1-0+2-1$ hot band is larger than the $0-0$ band. However, in 
light of the more recent experiments ${ }^{1}$ that agree with our calculations for the location of the ${ }^{2} \Pi$ state of $\mathrm{MgO}^{-}$, we do not attempt to develop an improved fit to the experimental data.

\section{CONCLUSIONS}

The computed spectroscopic constants for $\mathrm{MgO}^{-}$and $\mathrm{MgO}$ are in good agreement with experiment. The largest errors are in the position of the ${ }^{1} \Sigma^{+}$state with respect to the ${ }^{3} \Pi$ and ${ }^{1} \Pi$ states, and in the EA, which is slightly underestimated. The calculation show that the peaks at the lowest electron binding energy in the JHU spectra are due detachment from the ${ }^{2} \Pi$ state of $\mathrm{MgO}^{-}$to form the ${ }^{3} \Pi$ state. While detachment to form the ${ }^{1} \Sigma^{+}$state should occur at lower electron binding energies, it is not observed because it is a two electron process and is computed to have a very small probability of occurring.

\section{ACKNOWLEDGMENTS}

The authors acknowledge many helpful discussions with Kit Bowen and Hellen de Clercq. The authors would like to thank Kit Bowen for letting them reproduce his experimental spectra. 


\section{REFERENCES}

${ }^{1}$ J.H. Kim, X. Li, L.-S. Wang, H.L. de Clercq, C.A. Fancher, O.C. Thomas, K.H. Bowen, submitted to J. Phys. Chem. A.

${ }^{2}$ K.P. Huber, G. Herzberg "Constants of Diatomic Molecules", (Van Nostrand Reinhold, New York) 1979.

${ }^{3}$ P.C.F. Ip, K.J. Cross, R.W. Field, J. Rostas, B. Bourguignon, J. McCombie, J. Mol. Spect. 146 (1991) 409.

${ }^{4}$ P. Mürtz, H. Thümmel, C. Pfelzer, W. Urban, Mol. Phys. 86 (1995) 513.

${ }^{5}$ R.J. Gdanitz, R. Ahlrichs, Chem. Phys. Lett. 143 (1998) 413.

${ }^{6}$ H.-J. Werner and P.J. Knowles, J. Chem. Phys. 89 (1988) 5803 and H.-J. Werner and P.J. Knowles Chem. Phys. Lett. 145 (1988) 514.

${ }^{7}$ T.H. Dunning, J. Chem. Phys. 90 (1989) 1007.

${ }^{8}$ R.A. Kendall, T.H. Dunning, R.J. Harrison, J. Chem. Phys. 96 (1992) 6796.

${ }^{9}$ D.E. Woon, T.H. Dunning, J. Chem. Phys. 98 (1993) 1358.

${ }^{10}$ MOLPRO is a package of ab initio programs written by H.-J. Werner and P.J. Knowles, with contributions from J. Almlöf, R.D. Amos, A. Berning, D.L. Cooper, M.J.O. Deegan, A.J. Dobbyn, F. Eckert, S.T. Elbert, C. Hampel, R. Lindh, A.W. Llyod, W. Meyer, A. Nicklass, K. Peterson, R. Pitzer, A.J. Stone, P.R. Taylor, M.E. Mura, P. Pulay, M. Schütz, H. Stoll, and T. Thorseinsson.

${ }^{11}$ MOLECULE-SWEDEN is an electronic structure program written by $\mathrm{J}$. Almlöf, C.W. Bauschlicher, M.R.A. Blomberg, D.P. Chong, A. Heiberg, S.R. Langhoff, P.A. Malmqvist, A.P. Rendell, B.O. Roos, P.E.M. Siegbahn, P.R. Taylor.

${ }^{12}$ H. Thümmel, R. Klotz, S.D. Peyerimoff, Chem. Phys. 129 (1989) 417. 
${ }^{13}$ G.L. Gutzev, M. Nooijen, R.J. Bartlett, Chem. Phys. Lett. 276 (1997) 13. 


\section{TABLES}

TABLE I. Summary of the computed spectroscopic constants.

\begin{tabular}{lllll}
\hline \hline State & $r_{e}(\AA)$ & $\omega_{e}\left(\mathrm{~cm}^{-1}\right)$ & $T_{e}\left(\mathrm{~cm}^{-1}\right)$ & $T_{e}^{\prime}\left(\mathrm{cm}^{-1}\right)$
\end{tabular}

This work

$\begin{array}{lllrr}\mathrm{MgO}^{-2} \Sigma^{+} & 1.801 & 739 & 0 & \\ \mathrm{MgO}^{-2} \Pi & 1.970 & 512 & 4850 & \\ \mathrm{MgO}^{1} \Sigma^{+} & 1.771 & 760 & 12561 & 0 \\ \mathrm{MgO}^{3} \Pi & 1.888 & 645 & 14327 & 1766 \\ \mathrm{MgO}^{1} \Pi & 1.884 & 654 & 15182 & 2621\end{array}$

Experiment ${ }^{a}$

$\begin{array}{ccccc}\mathrm{MgO}^{-2} \Sigma^{+} & 1.794 & 670 \pm 80 & 0 & \\ \mathrm{MgO}^{-2} \Pi & 1.911 & 593 \pm 80 & 4800 & \\ \mathrm{MgO}^{1} \Sigma^{+} & (1.749) & 780 \pm 40 & 13240 & 0 \\ \mathrm{MgO}^{3} \Pi & 1.864 & 600 \pm 80 & 15760 \pm 140 & 2520 \pm 140 \\ \mathrm{MgO}^{1} \Pi & 1.854 & 650 \pm 80 & 16640 \pm 150 & 3400 \pm 150\end{array}$

Experiment $^{b}$

\begin{tabular}{lllr}
$\mathrm{MgO}^{1} \Sigma^{+}$ & 1.749 & 785 & 0 \\
$\mathrm{MgO}^{3} \Pi$ & 1.869 & 650 & 2620 \\
$\mathrm{MgO}^{1} \Pi$ & 1.864 & 664 & 3563 \\
\hline
\end{tabular}

${ }^{a}$ Reference 1. The ${ }^{1} \Sigma^{+} r_{e}$ was taken from Reference 2. The experimental results are $T_{0}$ not $T_{e}$ values.

${ }^{b}$ Reference 2 is used for the ${ }^{1} \Sigma^{+}$and ${ }^{1} \Pi$ states, and reference 4 is used for the ${ }^{3} \Pi$ state. 
TABLE II. Electron detachment overlaps.

\begin{tabular}{lrr}
\hline \hline & \multicolumn{2}{c}{ Ion State } \\
\cline { 2 - 3 } Neutral & ${ }^{2} \Sigma^{+}(\mathrm{r}=1.80 \AA)$ & ${ }^{2} \Pi(\mathrm{r}=1.97 \AA)$ \\
${ }^{1} \Sigma^{+}$ & 0.645 & 0.001 \\
${ }^{1} \Pi$ & 0.575 & 0.418 \\
${ }^{3} \Pi$ & 1.810 & 1.245 \\
\hline \hline
\end{tabular}


TABLE III. Computed Franck-Condon factors and Franck-Condon factors times the overlap of the wave functions.

\begin{tabular}{|c|c|c|c|c|c|c|c|}
\hline \multirow[b]{2}{*}{$\begin{array}{c}v(\text { ion }) \\
1 \Sigma^{+}\end{array}$} & \multirow[b]{2}{*}{$v$ (neutral) } & \multicolumn{3}{|c|}{${ }^{2} \Sigma^{+}$} & \multicolumn{3}{|c|}{${ }^{2} \Pi$} \\
\hline & & $\mathbf{E}(\mathrm{eV})$ & FC & FC*ovl & $\mathbf{E}(\mathrm{eV})$ & FC & FC*oul \\
\hline o & 0 & 1.643 & 0.910 & 0.587 & 1.056 & 0.028 & 0.000 \\
\hline $\mathbf{0}$ & 1 & 1.736 & 0.088 & 0.057 & 1.149 & 0.114 & 0.000 \\
\hline $\mathbf{0}$ & 2 & 1.828 & 0.002 & 0.001 & 1.241 & 0.214 & 0.000 \\
\hline 0 & 3 & 1.919 & 0.000 & 0.000 & 1.332 & 0.247 & 0.000 \\
\hline 0 & 4 & 2.009 & 0.000 & 0.000 & 1.421 & 0.199 & 0.000 \\
\hline 1 & 0 & 1.553 & 0.082 & 0.053 & 0.993 & 0.084 & 0.000 \\
\hline 1 & 1 & 1.646 & 0.746 & 0.481 & 1.086 & 0.179 & 0.000 \\
\hline 1 & 2 & 1.738 & 0.167 & 0.108 & 1.178 & 0.118 & 0.000 \\
\hline 1 & 3 & 1.829 & 0.005 & 0.003 & 1.269 & 0.008 & 0.000 \\
\hline 1 & 4 & 1.919 & 0.000 & 0.000 & 1.359 & 0.037 & 0.000 \\
\hline 2. & 0 & 1.464 & 0.007 & 0.005 & 0.932 & 0.137 & 0.000 \\
\hline 2 & 1 & 1.558 & 0.144 & 0.093 & 1.025 & 0.126 & 0.000 \\
\hline 2 & 2 & 1.650 & 0.601 & 0.387 & 1.117 & 0.003 & 0.000 \\
\hline 2 & 3 & 1.740 & 0.238 & 0.153 & 1.208 & 0.066 & 0.000 \\
\hline${ }^{2} \pi$ & 4 & 1.830 & 0.011 & 0.007 & 1.297 & 0.113 & 0.000 \\
\hline 0 & 0 & 2.078 & 0.511 & 0.294 & 1.491 & 0.525 & 0.220 \\
\hline o & 1 & 2.158 & 0.316 & 0.182 & 1.571 & 0.342 & 0.143 \\
\hline 0 & 2 & 2.237 & 0.122 & 0.070 & 1.650 & 0.108 & 0.045 \\
\hline 0 & 3 & 2.315 & 0.038 & 0.022 & 1.728 & 0.022 & 0.009 \\
\hline 0 & 4 & 2.393 & 0.010 & 0.006 & 1.805 & 0.003 & 0.001 \\
\hline 1 & 0 & 1.988 & 0.372 & 0.214 & 1.428 & 0.325 & 0.136 \\
\hline 1 & 1 & 2.068 & 0.058 & 0.033 & 1.508 & 0.059 & 0.025 \\
\hline 1 & 2 & 2.147 & 0.246 & 0.142 & 1.587 & 0.317 & 0.133 \\
\hline 1 & 3 & 2.225 & 0.188 & 0.108 & 1.665 & 0.213 & 0.089 \\
\hline 1 & 4 & 2.302 & 0.088 & 0.051 & 1.743 & 0.069 & 0.029 \\
\hline 2 & 0 & 1.899 & 0.103 & 0.059 & 1.367 & 0.113 & 0.047 \\
\hline 2 & 1 & 1.979 & 0.373 & 0.215 & 1.447 & 0.278 & 0.116 \\
\hline 2 & 2 & 2.058 & 0.004 & 0.002 & 1.526 & 0.010 & 0.004 \\
\hline 2 & 3 & 2.137 & 0.116 & 0.067 & 1.604 & 0.167 & 0.070 \\
\hline${ }^{2} \pi$ & 4 & 2.214 & 0.183 & 0.105 & 1.681 & 0.256 & 0.107 \\
\hline 0 & 0 & 1.961 & 0.479 & 0.868 & 1.373 & 0.561 & 0.703 \\
\hline 0 & 1 & 2.039 & 0.324 & 0.587 & 1.452 & 0.330 & 0.413 \\
\hline 0 & 2 & 2.117 & 0.134 & 0.243 & 1.529 & 0.091 & 0.115 \\
\hline 0 & 3 & 2.193 & 0.044 & 0.080 & 1.606 & 0.018 & 0.020 \\
\hline 0 & 4 & 2.269 & 0.013 & 0.023 & 1.682 & 0.002 & 0.003 \\
\hline
\end{tabular}




\begin{tabular}{llllllll}
1 & 0 & 1.871 & 0.382 & 0.691 & 1.311 & 0.311 & 0.390 \\
1 & 1 & 1.949 & 0.035 & 0.064 & 1.389 & 0.092 & 0.115 \\
1 & 2 & 2.027 & 0.228 & 0.412 & 1.467 & 0.341 & 0.428 \\
1 & 3 & 2.103 & 0.197 & 0.356 & 1.543 & 0.193 & 0.242 \\
1 & 4 & 2.179 & 0.100 & 0.181 & 1.619 & 0.053 & 0.066 \\
2 & 0 & 1.782 & 0.119 & 0.216 & 1.250 & 0.099 & 0.124 \\
2 & 1 & 1.861 & 0.348 & 0.631 & 1.328 & 0.295 & 0.370 \\
2 & 2 & 1.938 & 0.016 & 0.029 & 1.406 & 0.001 & 0.001 \\
2 & 3 & 2.015 & 0.088 & 0.159 & 1.482 & 0.217 & 0.272 \\
2 & 4 & 2.090 & 0.176 & 0.319 & 1.558 & 0.254 & 0.318 \\
\hline \hline
\end{tabular}




\section{FIGURES}

FIG. 1. The JHU photoelectron spectra of $\mathrm{MgO}^{-}$.

FIG. 2. A simulation of the photoelectron spectra of $\mathrm{MgO}^{-}$. The various components are shown below. The formation of the ${ }^{1} \Sigma^{+}$is shown as a dashed line, the ${ }^{1} \Pi$ as a dotted line and the ${ }^{3} \Pi$ as a chained line. 
Electron Counts

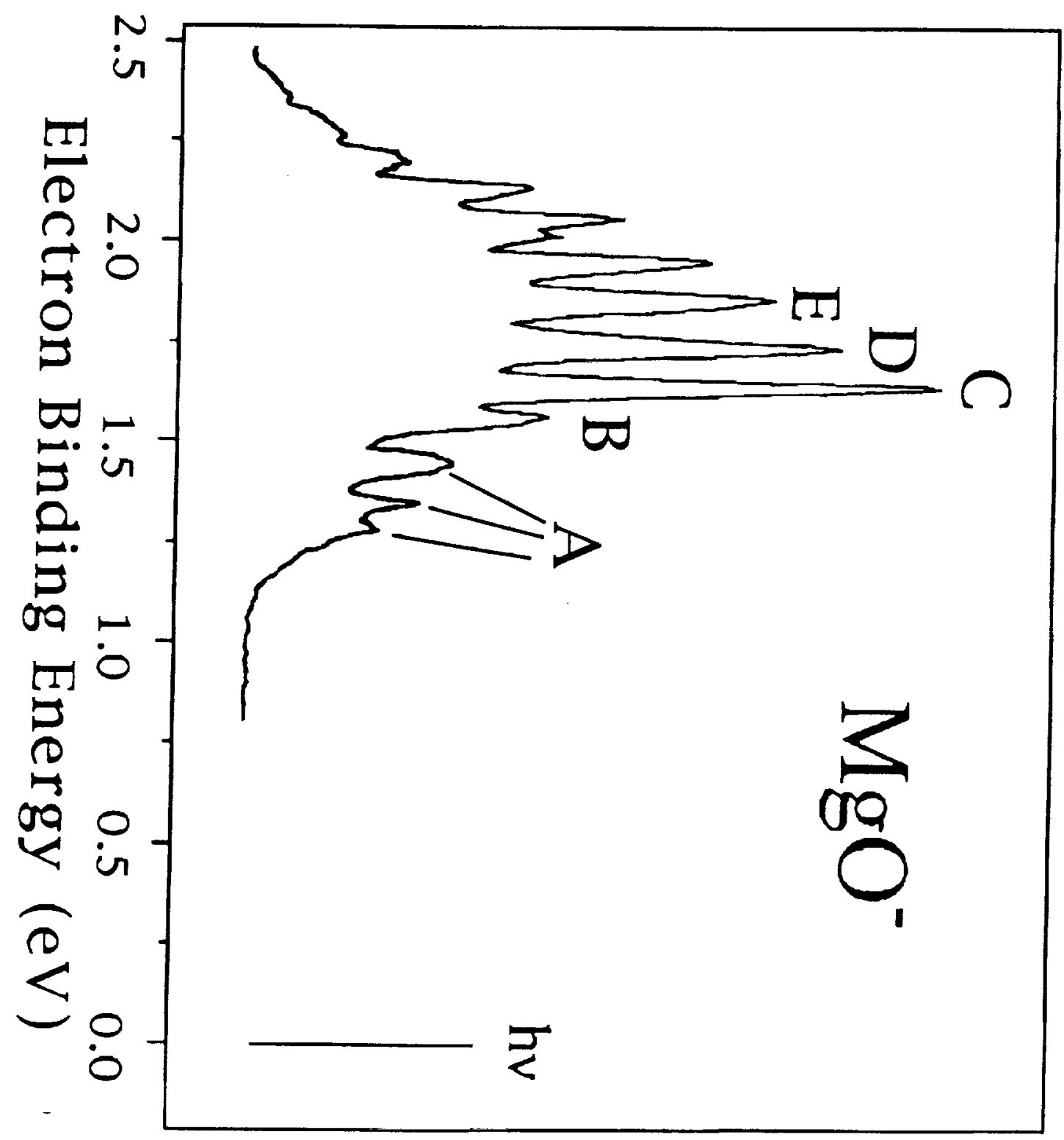




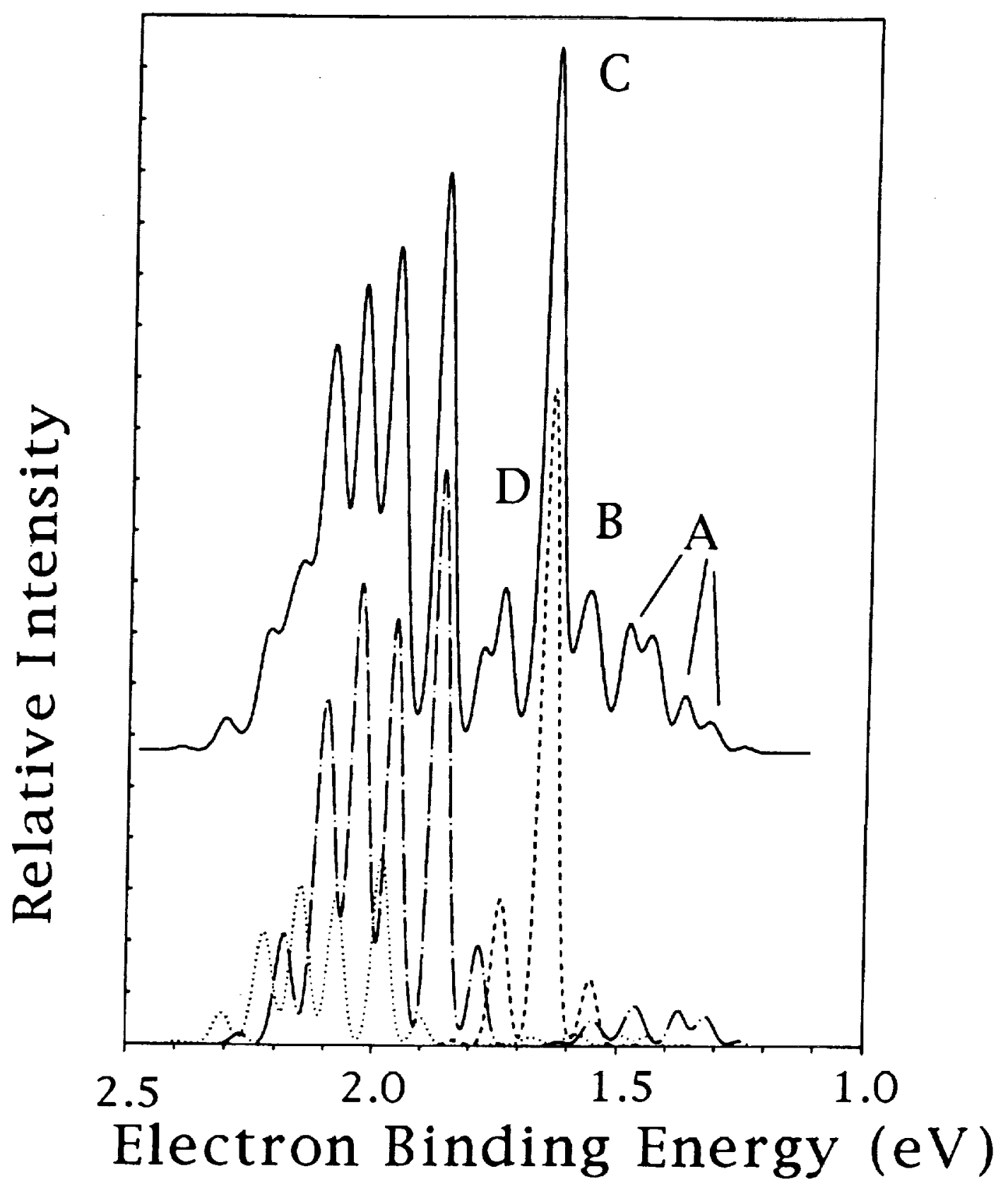

